\title{
INFLUENCE OF RECTANGULAR STRIPS' SIZE ON AERODYNAMIC PERFORMANCE OF A HIGH-SPEED TRAIN SUBJECTED TO CROSSWIND
}

\author{
Mengying Wang ${ }^{1,2}$, Zhenxu Sun ${ }^{1, *}$, Shengjun $\mathrm{Ju}^{1}$, Guowei Yang ${ }^{1,2}$ \\ 1. Key Laboratory for Mechanics in Fluid Solid Coupling Systems, Institute of Mechanics, Chinese \\ Academy of Sciences, Beijing, 100190, China \\ 2. School of Engineering Sciences, University of Chinese Academy of Sciences, Beijing, 101408, China \\ * Corresponding author. Email: sunzhenxu@imech.ac.cn
}

\begin{abstract}
Conventional studies usually assume that the train surface is smooth, so as to simplify the numerical calculation. In fact, the surface of the train is irregular, which will change the flow characteristics in the boundary layer and further affect the aerodynamic performance of a train. In this work, roughness is applied to the roof of a 1:25 scaled train model in the form of longitudinal strips. Firstly, the improved delayed detached eddy simulation (IDDES) method is adopted to simulate the aerodynamic performance of the train model with both smooth and rough surface, which are subjected to crosswind. Results show that the side force coefficient and the roll moment coefficient subjected to rough model decreased by $3.71 \%$ and $10.56 \%$ compared with the smooth model. Then, the width, height and length of the strips are selected as variables to design different numerical simulation schemes based on the orthogonal experimental design method. Through variance analysis, it can be found that four design parameters have no significant effect on the side force coefficient. Meanwhile, for the roll moment coefficient, the length of the strips in the straight region of the train has a significant effect and the width of the strips has a highly significant effect on it. These conclusions can provide a theoretical basis to improve the aerodynamic performance of the high-speed train subjected to crosswind.
\end{abstract}

Keywords: High-speed train, Crosswind, Rectangular strips, Aerodynamic performance, Orthogonal design, Variance analysis

\section{INTRODUCTION}

With the continuous development of railway transportation, the aerodynamic problems of high-speed trains are more prominent. When a high-speed train is subjected to crosswind, the aerodynamic performance will be deteriorated, and can even cause a rollover accident. For example, in 1981, a passenger train in India fell to the bottom of a bridge under the influence of a hurricane, resulting in more than 800 deaths. Therefore, it is particularly important to systematically study the aerodynamic characteristics of a train under crosswind. Numerical simulation has been adopted in various industrial fields $[1,2,3]$, and is one of the main research methods for high-speed train aerodynamics, which owns the advantages of low cost and high efficiency. Ben [4] obtained the aerodynamic lift of a high-speed train subjected to crosswind with different angles through numerical simulations. Justin et al. [5] used different turbulence models to simulate the flow field around a train subjected to crosswind. Liu et al. [6] conducted a study on the critical overturning wind speed of a high-speed train based on the numerical results of aerodynamic forces and torques. Gong et al. [7] established a three-dimensional viaduct-train coupling model, and performed a numerical simulation when it was subjected to the coupled effect of train speed and wind speed.

According to the basic crosswind aerodynamic characteristics of a high-speed train, scholars begin to explore applicable optimization methods. At present, most of the commonly used methods are carried out from the macro-scale, such as the shape design of the head of the train. However, due to many limitations in actual engineering, the benefits of such methods are limited. Therefore, some scholars try to start from a microscopic point of view to explore a new way to improve the aerodynamic performance of a high-speed train. Wang et al. [8,9] designed and studied the noise reduction effects of different types of microstructured surfaces. Miao et al. [10] found that adding ribs on the top of the train can help reduce the surface pressure. Sun [11] analyzed how the design parameters of different types of microstructure units influence the drag reduction effect of highspeed trains. Zhu et al. [12] and Tang et al. [13] studied the drag reduction mechanisms of non-smooth surface with ball sockets and convex hulls, respectively. Based on the existing research, the microstructures are mainly used for drag and noise reduction of high-speed trains. However, there is no relevant references 
about how the microstructures affect the crosswind aerodynamic performance of high-speed trains. Browand et al. [14] had mentioned in their study that design factors may have a strong influence on the stability of train bodies. Therefore, it is an important subject to evaluate the influence of the design parameters of the microstructure on the crosswind stability of high-speed trains.

Experiment is often used to explore and confirm the laws of science. However, with the rapid development of science and technology, more and more factors are involved in the experiment, and the relationship between the factors becomes more and more complicated. The expected goal cannot be obtained by experience easily, so the concept of experimental design has emerged. There are many experimental design methods, among which orthogonal experimental design has been widely used. For example, Fan et al. [15] took the total inlet pressure, power split, working mode and throttle lever position as the experimental factors to evaluate the steady-state performance of engine by using the orthogonal experimental design. Cheng et al. [16] took the inlet width, the axial length of the blade, and the axial distance between the blade and outlet as the experimental factors to optimize the shape of the well-used submersible pump space guide vane on the base of the orthogonal design method.

In this paper, the 1:25 scale model of the 390 Pendolino train is used as the prototype, and the non-smooth surface is designed by adding a rectangular strip on the top of the leading car. Section 2 firstly introduces the numerical algorithm, computational models, computational domain and boundary conditions adopted in this study. And numerical validation has also been performed in this section to ensure the correctness of the numerical algorithm and the computational mesh. Section 3 adopts the method of combining orthogonal experimental design and variance analysis to analyze the sensitivity between the design parameters of the rectangular strip and the train aerodynamic force/torque. Finally, Section 4 concludes the research by listing the main research outcomes along with providing suggestions for future work.

\section{NUMERICAL SIMULATION 2.1 Numerical Algorithm}

In this paper, commercial software STAR-CCM+ is used to study the aerodynamics of a high-speed train subjected to crosswinds. This software can guarantee high computational efficiency and make results converge easily, so it is widely used in many industrial fields. And the governing equations used in this study is three-dimensional incompressible N-S equations, which needs to be discretized by finite volume method. For the temporal discretization, this paper adopts the first-order implicit scheme, which can quickly obtain the convergent results. Meanwhile, in order to improve the calculation accuracy, for the spatial discretization, the second-order central difference scheme and the second-order upwind scheme are used to discretize the viscous and inviscid terms of the governing equations. In addition, the improved delayed detached eddy simulation (IDDES) method based on SST k- $\omega$ two-equation model will be used to simulate the flow field around the high-speed train with smooth and rough surfaces.

The detached eddy simulation (DES) method was proposed by Spalart et al. [17] in 2014. As a combination of the large eddy simulation (LES) method and Reynolds-averaged NavierStokes (RANS) method, DES can reduce calculation time while ensuring calculation accuracy. This method is mostly used to solve the problem of large-scale separation flow, but it is not universal. There are still some problems, such as the sensitivity of the grid, which will lead to the appearance of non-physical separation. To solve these problems, Mikhail et al. [18] proposed the delayed detached eddy simulation (DDES) method, that is, adding a delay function to the DES, which slows down the conversion from RANS to LES.

However, when use DDES to simulate, the slopes of the logarithmic regions solved by RANS and LES are different, which will interfere with the separation flow that is greatly affected by the boundary layer. Therefore, the improved delayed detached eddy simulation (IDDES) method was proposed, which can effectively process the grids solved by RANS and LES in the logarithmic area, and greatly reduce the grid correlation. This method has been widely used in the study of cross-wind stability of high-speed trains. For example, Munoz et al. [19] used this method to simulate the flow field around a high-speed train subjected to crosswind. Through analysis, they found that this method can capture the small vortex in the shear layer. Li et al. [20] obtained the surface pressure and the aerodynamic forces of a high-speed train subjected to crosswind by this method, which are in good agreement with the experimental results, indicating that this method can accurately predict the average flow field around the train.

\subsection{Computational Models and Computational Domain}

The smooth model is a 1:25 scale model of a class 390 Pendolino train, including a leading car and a semi-trailing car, as shown in Figure 1. For the convenience of analysis, the bogies, air conditioner, pantograph and windshields are being simplified without affecting the calculation results. The calculation cannot run indefinitely, so a computational domain needs to be determined, as shown by the black frame in Figure 1. It can be seen that the calculation model also includes a track and a splitter plate, which are about $0.3 \mathrm{~m}$ from the ground to avoid the influence from the boundary layer of the ground.

By adding rectangular strips to the two regions on the top of the leading car, a rough model is obtained, as shown in Figure 2. There are 15 strips in each region, and the distance between two adjacent strips is equal. Figure 3 shows the geometry of the rectangular strips. All strips have same cross-sectional area, but the length of the strips in different regions is different. The length of the strips in region 1 is $0.12 \mathrm{~m}$, and the length of the strips in region 2 is $0.24 \mathrm{~m}$. 

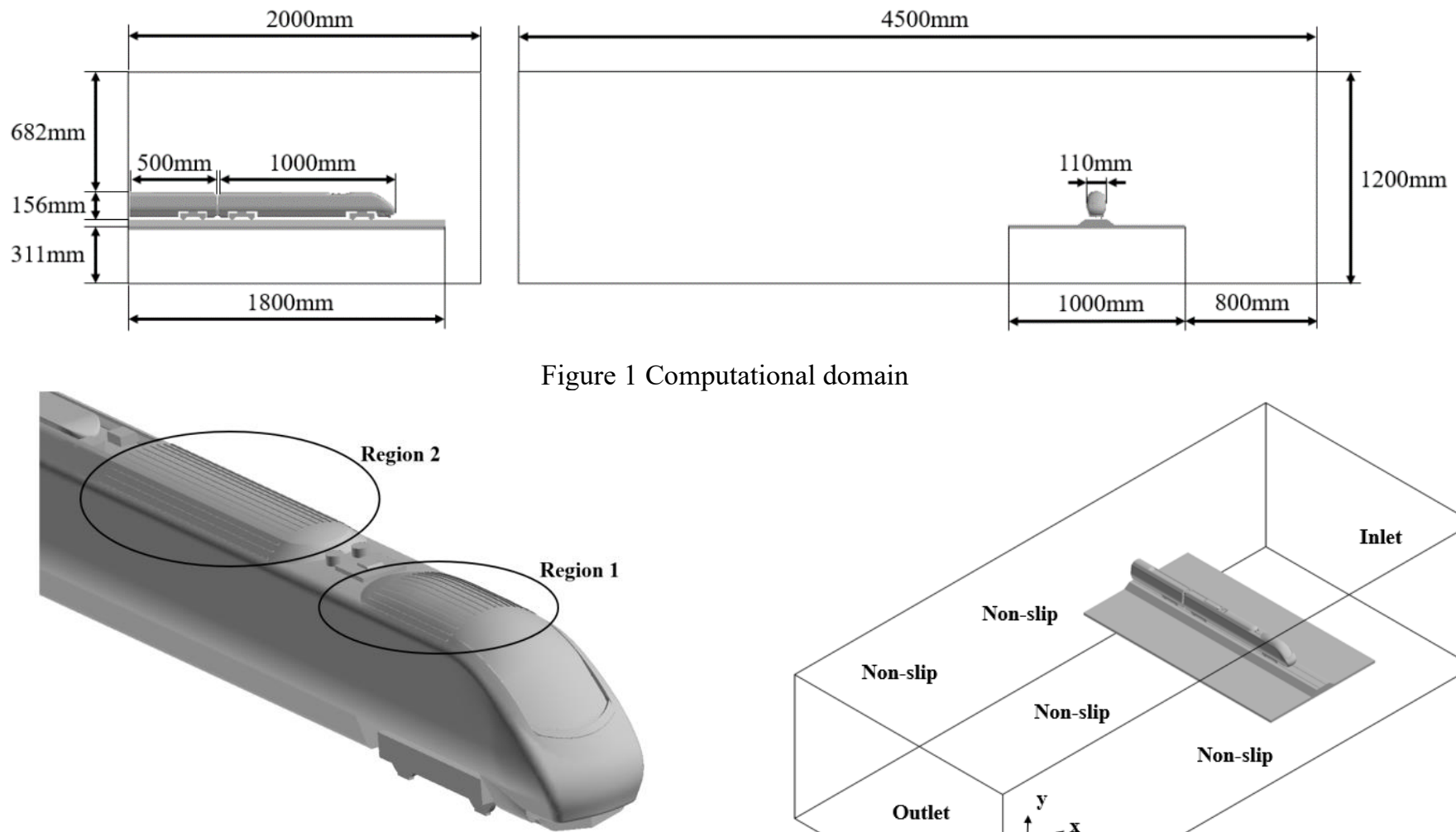

Figure 2 Computational model with rough surface

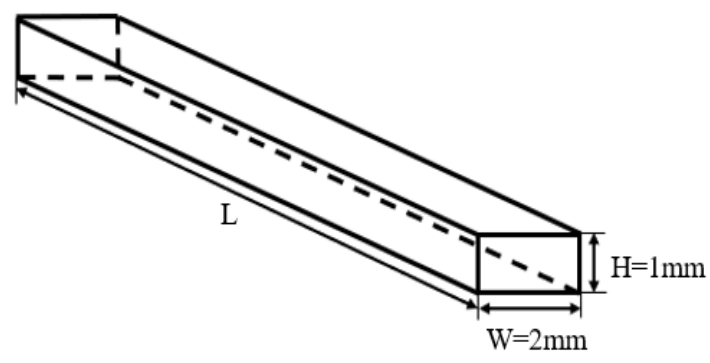

Figure 3 Geometry of the rectangular strip

\subsection{Boundary Conditions}

The boundary conditions are shown in Figure 4. Except for the inlet and outlet boundaries, non-slip wall conditions are specified at the other boundaries. A uniform airflow with a speed of $7.2 \mathrm{~m} / \mathrm{s}$ acts on the inlet boundary, while the outlet boundary has a zero-pressure condition. During the calculation, the computational model is stationary and is subjected to a relative wind with a yaw angle of $90^{\circ}$.

Figure 5 Position of all loops [19] 
In this paper, cartesian grids are used for spatial meshing and two sets of grids are generated, namely the coarse set and the fine set. The amount of grids in the former is 52.16 million, while the amount of grids in the latter is 88.87 million. Figure 6 gives the comparison of numerical and experimental results of pressure coefficient on the loops E and G. It can be seen from the figure that the distribution of pressure coefficients obtained by using different grid schemes is consistent, but when comparing with the experimental results, it is obvious that the scheme with fine grids has higher calculation accuracy. Therefore, all subsequent calculations are meshed in a manner consistent with fine grids.

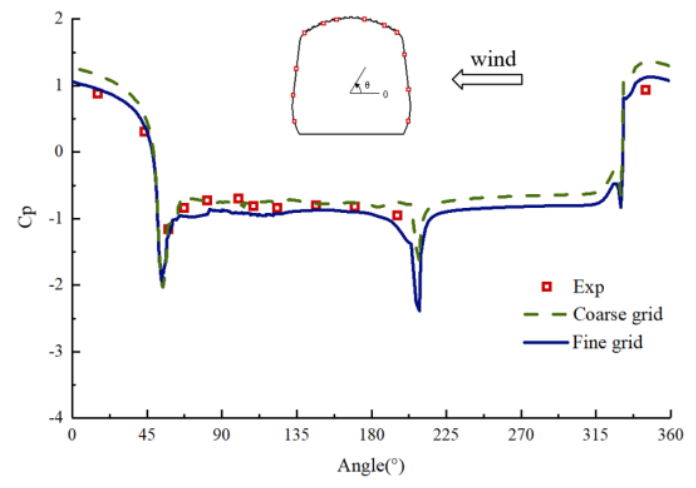

(a)

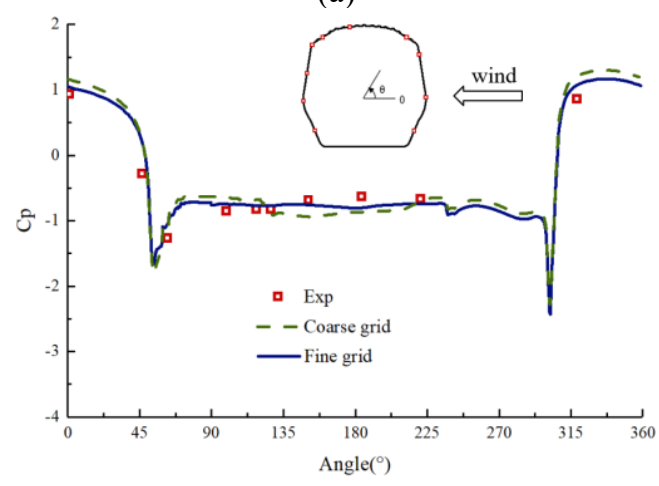

(b)

Figure 6 The numerical and experimental results of pressure coefficient on the loops E and G: (a) Loop E; (b) Loop G

Figures 7(a) and 7(b) show the grid distribution on the sections of $\mathrm{x}=0 \mathrm{~m}$ and $\mathrm{z}=-0.2 \mathrm{~m}$, respectively. It can be seen that three grid refinement regions A2, A3, A4 are set around the model, and the grid size is gradually increased. On this basis, two regions with rectangular strips are further refined to improve the calculation accuracy, as shown in area A1. In addition, considering the influence of the boundary layer effect, prism layer grids are generated on the surface of the train and strips. To achieve natural transition between grids, a total of 10 prism layers are set up and the growth ratio is set at 1.1. This sizing gives an average of nondimensional wall distance, $\mathrm{y}^{+}$, of approximately 1.13 . And it can be seen from the region $\mathrm{P}$ in
Figure $7(\mathrm{c})$ that the strips' geometry has not been damaged, indicating that the adopted grid scheme is of high quality.
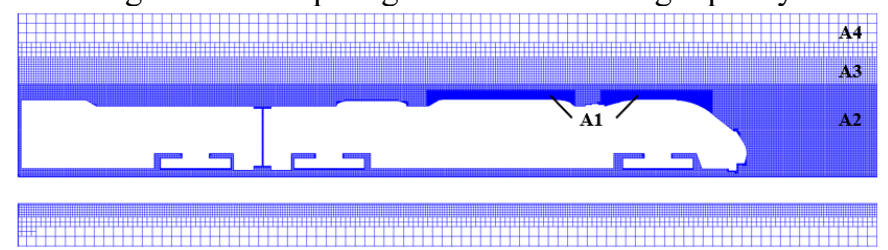

(a)

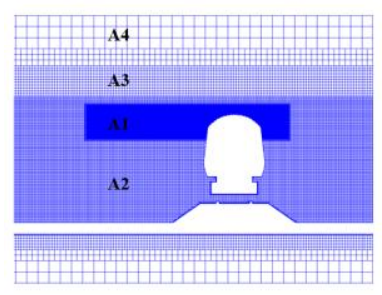

(b)

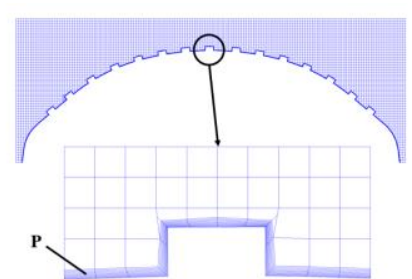

(c)
Figure 7 Computational mesh

\subsection{Characteristics of Aerodynamic Loads}

Side force and roll moment are important indicators for evaluating the stability of high-speed trains subjected to crosswind. To simplify analysis, define side force coefficient $C_{s}$ and roll moment coefficient $C_{m z}$ as follows:

$$
C_{s}=\frac{F_{s}}{0.5 \rho V_{r e f}^{2} A_{r e f}}, \quad C_{m z}=\frac{M_{z}}{0.5 \rho V_{r e f}^{2} A_{r e f} H_{r e f}}
$$

where $F_{s}$ is the side force, $M_{z}$ is the roll moment, $A_{r e f}=0.1232 \mathrm{~m}^{2}$ [22] is the projected area of the train in the x direction, $H_{r e f}$ is the train carriage height. Figure 8 gives the results of $C_{s}$ and $C_{m z}$ of different models. By calculating the relative error, $C_{s}$ and $C_{m z}$ of rough model are decreased by $3.71 \%$ and $10.56 \%$ compared with smooth model, indicating that the train with certain roughness can operate more safely and stably.

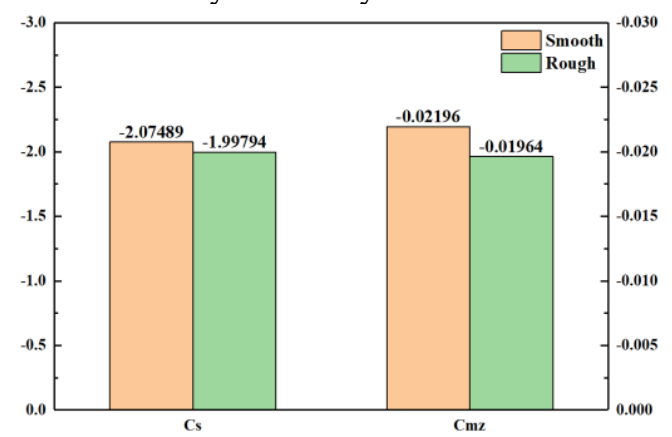

Figure 8 Results of $C_{s}$ and $C_{m z}$ of smooth surface model and rough surface model

\section{RESULTS OF SENSITIVITY ANALYSIS}

The design of experiments (DOE) is a statistical method for scientific arrange multi-factor experiments, which spreads the limited number of design samples in the whole design space [23]. There are many design methods including orthogonal experimental design, full factorial design, central composite design and Latin hypercube experiment. The variance analysis technique as a statistic method can be used to analyze the 
difference between the experimental results under different observation conditions [24, 25].

In the following discussion of sensitivity analysis, four design variables of rectangular strips aforementioned have been considered, namely L1, L2, W and $\mathrm{H}$. Each design variance has three levels, i.e. $\mathrm{L} 1 \in\{0.06,0.09,0.12\}, \mathrm{L} 2 \in\{0.12,0.18,0.24\}$, $\mathrm{W} \in\{0.001,0.003,0.005\}, \mathrm{H} \in\{0.0003,0.00075,0.0012\}$. The design variable experimental arrangement and the force characteristics of numerical simulation are shown in Table 1. Design variables are arranged in the first four columns of the orthogonal experimental design table $\mathrm{L}_{18}\left(3^{8}\right)$, and the other four columns are allotted to random errors. For all design schemes, the same settings as in section 2 are used for numerical calculations.

Table 2 and Table 3 show the results of analysis of variance of the side force coefficient and the roll moment coefficient, respectively. ' $\mathrm{df}$ ' denotes the freedom degree, ' $\mathrm{F}$ ' is the ratio of the sum of squares of the mean deviation of each factor to that of error and 'Sig.' is the statistical significance used to estimate the possibility that the result occurs. The confidence interval is often set to be 0.01 or 0.05 in the variance analysis process of the experimental data.
In Table 2, it is observed that for side force coefficient, the Sig.s of the length of rectangular strips in region 1 (L1), the length of rectangular strips in region 2 (L2), the width of rectangular strips $(\mathrm{W})$ and the height of rectangular strips $(\mathrm{H})$ are more than 0.05 , which implies that the effects of all design variables are not substantial. The results of between-subjects effects for roll moment coefficient are shown in Table 3. It is observed that for roll moment coefficient, the Sig.s of the length of rectangular strips in region 2 (L2) and the height of rectangular strips $(\mathrm{H})$ are less than 0.05 , and this implies that the effects of L2 and $\mathrm{H}$ on the roll moment are substantial, but the effects of the other two design variables, namely L1 and $\mathrm{W}$ are not obvious. What's more, the Sig. of the height of rectangular strips $(\mathrm{H})$ is less than 0.01 , which means the effects of $\mathrm{H}$ on the roll moment is highly substantial.

As a summary of these results shown, the values of the length of rectangular strips in region 2 (L2) and the height of rectangular strips $(\mathrm{H})$ must be investigated first, while the values of the length of rectangular strips in region 1 (L1) and the width of rectangular strips $(\mathrm{W})$ could be neglected in the design process of the aerodynamic performance of a high-speed train subjected to crosswind.

Table 1 Experimental design of the rectangular strips' size

\begin{tabular}{|c|c|c|c|c|c|c|}
\hline \multirow{2}{*}{ Test number } & \multicolumn{4}{|c|}{ Design variable } & \multicolumn{2}{|c|}{ Aerodynamic coefficient } \\
\hline & $\mathrm{L} 1 / \mathrm{m}$ & $\mathrm{L} 2 / \mathrm{m}$ & $\mathrm{W} / \mathrm{m}$ & $\mathrm{H} / \mathrm{m}$ & $C_{s}$ & $C_{m z}$ \\
\hline 1 & 0.06 & 0.12 & 0.001 & 0.0003 & -2.04473 & -0.01938 \\
\hline 2 & 0.06 & 0.18 & 0.003 & 0.00075 & -2.01962 & -0.02082 \\
\hline 3 & 0.06 & 0.24 & 0.005 & 0.0012 & -2.03986 & -0.02128 \\
\hline 4 & 0.09 & 0.12 & 0.001 & 0.00075 & -2.14190 & -0.01805 \\
\hline 5 & 0.09 & 0.18 & 0.003 & 0.0012 & -2.02992 & -0.01851 \\
\hline 6 & 0.09 & 0.24 & 0.005 & 0.0003 & -2.04308 & -0.02089 \\
\hline 7 & 0.12 & 0.12 & 0.003 & 0.0003 & -2.02131 & -0.02074 \\
\hline 8 & 0.12 & 0.18 & 0.005 & 0.00075 & -2.08779 & -0.02114 \\
\hline 9 & 0.12 & 0.24 & 0.001 & 0.0012 & -2.05464 & -0.01628 \\
\hline 10 & 0.06 & 0.12 & 0.005 & 0.0012 & -2.08355 & -0.01331 \\
\hline 11 & 0.06 & 0.18 & 0.001 & 0.0003 & -1.99375 & -0.02289 \\
\hline 12 & 0.06 & 0.24 & 0.003 & 0.00075 & -2.01262 & -0.02229 \\
\hline 13 & 0.09 & 0.12 & 0.003 & 0.0012 & -2.06636 & -0.01684 \\
\hline 14 & 0.09 & 0.18 & 0.005 & 0.0003 & -2.00524 & -0.02429 \\
\hline 15 & 0.09 & 0.24 & 0.001 & 0.00075 & -2.00941 & -0.02317 \\
\hline 16 & 0.12 & 0.12 & 0.005 & 0.00075 & -2.04666 & -0.01876 \\
\hline 17 & 0.12 & 0.18 & 0.001 & 0.0012 & -2.02588 & -0.01678 \\
\hline
\end{tabular}




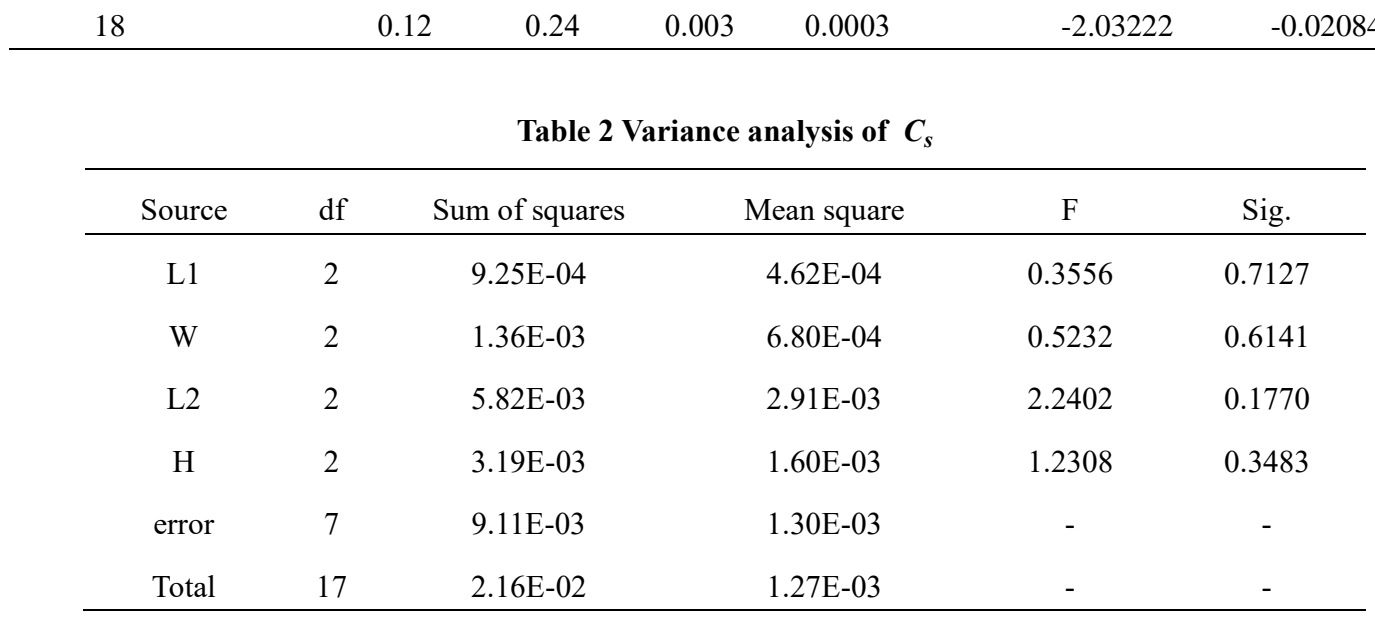

Table 3 Variance analysis of $C_{m z}$

\begin{tabular}{cccccc}
\hline Source & df & Sum of squares & Mean square & F & Sig. \\
\hline L1 & 2 & $4.69 \mathrm{E}-06$ & $2.35 \mathrm{E}-06$ & 0.8117 & 0.4819 \\
W & 2 & $1.23 \mathrm{E}-06$ & $6.13 \mathrm{E}-07$ & 0.2122 & 0.8138 \\
L2 & 2 & $3.41 \mathrm{E}-05$ & $1.70 \mathrm{E}-05$ & 5.8939 & 0.0316 \\
H & 2 & $6.39 \mathrm{E}-05$ & $3.20 \mathrm{E}-05$ & 11.0588 & 0.0068 \\
error & 7 & $2.02 \mathrm{E}-05$ & $2.89 \mathrm{E}-06$ & - & - \\
Total & 17 & $1.34 \mathrm{E}-04$ & $7.87 \mathrm{E}-06$ & - & - \\
\hline
\end{tabular}

\section{CONCLUSION}

In this paper, the influence of rectangular strips on the aerodynamic performance of a high-speed train subjected to crosswind are demonstrated by using IDDES method based on SST k- $\omega$ two-equation model, and the effects of rectangular strips' geometric parameters are analyzed by variance analysis method coupled with orthogonal experimental design. Main findings are summarized as follow:

(1) Compared with the smooth model, the rough model can reduce side force coefficient and roll moment coefficient by $3.71 \%$ and $10.56 \%$ respectively, thereby improving the crosswind stability of the train.

(2) It is observed that the variance analysis method can be used to accurately analyze the effects of rectangular strips' geometric parameters on the aerodynamic performance of a high-speed train subjected to crosswind. It can be applied to investigate and design the cross-wind control system of a highspeed train.

(3) In the design process of a high-speed train with rectangular strips, the values of the length of rectangular strips in region 2 (L2) and the height of rectangular strips (H) must be investigated first since small variance may lead to a substantial effect.

The above conclusions can provide a new idea for the subsequent crosswind aerodynamic optimization design of high- speed trains. However, current study mainly focuses on the scaled model. How the micro-structure surface affects the aerodynamic performance of high-speed trains in actual operation is still uncertain, which will be the main objective of our future research.

\section{ACKNOWLEDGEMENTS}

The work was financed by the program of China Scholarships Council and Youth Innovation Promotion Association CAS (2019020).

\section{REFERENCES}

[1] Luo Xianwu, Huang Renfang, Ji Bin, 2016. Transient cavitating vortical flows around a hydrofoil using k-omega partially averaged Navier-Stokes model. Modern Physics Letters B 30 (1).

[2] Luo Xianwu, Ye Weixiang, Huang Renfang, Wang Yiwei, Du Tezhuan, Huang Chenguang, 2020. Numerical investigations of the energy performance and pressure fluctuations for a waterjet pump in a non-uniform inflow. Renewable Energy 153, 1042-1052.

[3] Zhao Xiaoli, Sun Zhenxu, A new method for numerical simulation of two trains passing by each other at the same speed, Journal of Hydrodynamics, 2010, 22(5): 697-702.

[4] Ben Diedrichs. Aerodynamic Calculations of Crosswind Stability of a High-speed Train Using Control Volumes of 
Arbitrary Polyhedral Shape. BBAA VI International Colloquium on: Bluff Bodies Aerodynamics \& Applications. July, 20-24, 2008.

[5] Justin A. Morden, Hassan Hemida, Chris. J. Baker. Comparison of RANS and Detached Eddy Simulation Results to Wind-Tunnel Data for the Surface Pressures Upon a Class 43 High-Speed Train. Journal of Fluids Engineering. APRIL 2015, Vol. 137 / 041108-1

[6] Liu Rong, Yao Song, Xu Jiaoe. Study on critical overturning wind speed of high-speed train under cross wind $[\mathrm{J}]$. Journal of Railway Science and Engineering. 2019, 16(11): 2643-2650.

[7] Gong Yanjun, Huang Zundi, Chang Ning. Aerodynamic load analysis of intercity EMU car bodies operating on the viaduct under cross-wind environment[J]. Journal of Railway Science and Engineering. 2020, 17(11): 2748-2755.

[8] Wang Jiugen, Chen Shihong, Wang Qingjiu. Effect of bionic rhombic surface texture on frictional noise of high-speed train. Journal of Traffic and Transportation Engineering. 2014(02), 14(01).

[9] Wang Jiugen, Chen Shihong. Bionic Design of Koch Snowflake Surface Texture and Its Effects on Air Frictional Noise of High Speed Train [J]. Journal of Mechanical Engineering, 2014, 50(7): 78-83.

[10] X. Miao, G. Gao. Influence of ribs on train aerodynamic performances. Journal of Central South University,2015, 22(5), pp.1986-1993.

[11] Sun Pengpeng. Research on Aerodynamic Drag Reduction of High-speed Train with Non-smooth Surface[D]. Hangzhou: Zhejiang University, 2012.

[12] Zhu Haiyan, Zhang Yi, Zhao Huairui, Wu Pingbo, Shao Xiaofeng. Drag reduction technology of high-speed train based on boundary layer control. Journal of Traffic and Transportation Engineering. 2017(04). 17(02).

[13] Tang Kun, Ma Shuwen, Liang Hongqin, Ding Guofu. Simulation Research of the Drag Reduction of High-Speed Train Microstructured Surfaces [J]. Machinery Design \& Manufacture, 2020(09): 213-216.

[14] Browand, F., Ross, J., McCallen, R., 2009. The Aerodynamics of Heavy Vehicles II: Trucks, Buses, and Trains. Berlin.

[15] Fan Zebing, Zhang Xiangqian, Wang Shufu, Wang Fengming. Orthogonal design of aero-engine altitude simulation test[J]. Gas Turbine Experiment and Research, 2018, 31(04): 3741.

[16] Cheng Xiaorui, Zhang Xvelian. Hydraulic optimization of spatial guide vane on submersible well pump based on the orthogonal design[J]. Journal of Lanzhou University of Technology, 2020, 46(05): 61-67.

[17] P. R. Spalart, S. Deck, M. L. Shur, K. D. Squires, M. Kh. Strelets, A. Travin. A New Version of Detached-eddy Simulation, Resistant to Ambiguous Grid Densities[J]. Theoretical and Computational Fluid Dynamics, 2006, 20(3).

[18] Mikhail L. Shur, Philippe R. Spalart, Mikhail Kh. Strelets, Andrey K. Travin. A hybrid RANS-LES approach with
delayed-DES and wall-modelled LES capabilities[J]. International Journal of Heat and Fluid Flow, 2008, 29(6).

[19] J. Munoz-Paniagua, J. García, B. Lehugeur. Evaluation of RANS, SAS and IDDES models for the simulation of the flow around a high-speed train subjected to crosswind. Journal of Wind Engineering \& Industrial Aerodynamics. 171 (2017) 5066

[20] Tian Li. Comparisons of Shear Stress Transport and Detached Eddy Simulations of the Flow Around Trains. Journal of Fluids Engineering. NOVEMBER 2018, Vol. 140

[21] Hashmi S A, Hemida H, Soper D. Wind tunnel testing on a train model subjected to crosswinds with different windbreak walls[J]. Journal of Wind Engineering and Industrial Aerodynamics, 2019, 195.

[22] Dorigatti, F., Sterling, M., Baker, C., Quinn, A., 2015. Crosswind effects on the stability of a model passenger train - A comparison of static and moving experiments. J. Wind Eng. Ind. Aerod. 138, 36-51.

[23] Shengjun Ju, Chao Yan, Xiaoyong Wang, Yupei Qin, Zhifei Ye. Effect of energy addition parameters upon scramjet nozzle performances based on the variance analysis method[J]. Aerospace Science and Technology,2017.

[24] Wei Huang, Shi-bin Luo, Zhen-guo Wang, Parametric sensitivity analysis of scramjet nozzle configuration, J. Propuls. Technol. 30 (6) (2009) 691-695.

[25] Ju Shengjun, Yan Chao, Ye Zhifei, Multi-parametric sensitivity analysis of airbreathing hypersonic vehicle, J. Beijing Univ. Aeronaut. Astronaut. 43 (5) (2017) 927-935. 